\title{
Solid state fermentation for the production of lipolytic fungal enzymes
}

\author{
Diego ROMANO1*, Raffaella GANDOLFI² ${ }^{2}$, Roberto GUALANDRIS ${ }^{1}$, Andrea ROMANO ${ }^{1}$, Flavio STARACE ${ }^{1}$, Raffaella VILLA ${ }^{3}$, Francesco \\ MOLINARI ${ }^{1}$ \\ ${ }^{1}$ Dipartimento di Scienze e Tecnologie Alimentari e Microbiologiche, Universitá degli Studi di Milano, Via Celoria 2, 20133 Milano; \\ 2Istituto di Chimica Organica "Alessandro Marchesini", Università degli Studi di Milano, Via Venezian 21, 20133 Milano, Italy; \\ ${ }^{3}$ Centre for Resource Management and Efficiency, Building 40, School of Applied Sciences, Cranfield University, Bedford MK43 OAL, UK
}

Received 9 July 2007 / Accepted 17 September 2007

\begin{abstract}
The production of lypolitic moulds in solid state fermentation was studied. Aspergillus oryzae and Rhizopus oryzae were grown on rice hulls using different media. Tween 80 and olive oil were employed as main carbon sources; the whole solid cultures were lyophilised and employed in heptane for catalysing the formation of various geranyl esters with molar conversions ranging from 40 to $95 \%$, under optimised conditions, starting from $50 \mathrm{mM}$ geraniol and equimolar amount of the acid.
\end{abstract}

Key words: solid state fermentation, rice hulls, Rhizopus oryzae, Aspergillus oryzae, esterification, flavours.

\section{INTRODUCTION}

Solid-state fermentation (SSF) is a process occurring in the near-absence of free water employing an inert and/or natural solid support for the growth (Pandey et al., 1999). SSF may have considerable advantages over traditional liquid fermentations, such as easy aeration, low energy requirements, non sterile conditions, higher product yields and easier product recovery (Ooijkaas et al., 2000). A lot of published information concerning SSF regards the production of microbial metabolites and industrial enzymes; the interest has been mainly devoted to extracellular enzymes which are easily recovered and do not undergo major proteolytic degradation (Pandey et al., 2000).

Many different fungi have been grown on natural or synthetic impregnated supports, such as vermiculite (Silman et al., 1993), clay granules (Desgranges et al., 1993), amberlite (Gutierrez-Rojas et al., 1995), polyurethane foam (Hongzhang et al., 2006; John et al., 2007) or polystyrene (Zhu et al., 1994; Gautam et al., 2002). SSF can be carried out also on a variety of agricultural residues such as wheat straw, bagasse, corn cobs, rice hulls (Pandey et al., 2000), babassu cakes (Gutarra et al., 2007) and melon wastes (Alkan et al., 2007). Rice hulls are cheap waste-products and have been employed for the growth of Colletotrichum truncatum for use as mycoherbicide (Silman et al., 1991).

Aspergillus oryzae and Rhizopus oryzae are among the microorganisms traditionally studied in SSF, such as the Koji process or grown on polyurethane foam for the production of extracellular lipases (Cbristen et al., 1995), proteases (Qzawa et al., 1996) and amylases (Murado et al.,

* Corresponding author. Phone: +39 0250319134;

Fax: +39 0250319238; E-mail: diego.romano@unimi.it
1997). We have previously observed that strains of $A$. oryzae and $R$. oryzae produced extracellular and mycelium bound carboxylesterases with relevant activities and their dry mycelium efficiently and selectively catalysed the esterification between free acetic acid and primary alcohols in organic solvent (Molinari et al., 2000; Gandolfi et al., 2001). Acetic esters, such as geranyl acetate, are important flavour and fragrance components in the food and cosmetic industry (Longo and Sanroman, 2006).

In this work we have studied the growth and lypolitic activity of $A$. oryzae MIM in SSF employing rice hulls as support, since the mycelial morphology of these moulds seemed to be well suited for growing on solid supports. The carboxylesterase activity of the resulting biocatalyst has been exploited for the acylation of geraniol in organic solvent with acetic, butyric and hexanoic acid. The procedure developed with $A$. oryzae MIM was also applied to four other microbial strains, belonging to the species $R$. oryzae (namely $R$. oryzae CBS $112.07, R$. oryzae CBS 260.28, $R$. oryzae CBS 328.47, $R$. oryzae CBS 391.34).

\section{MATERIALS AND METHODS}

Microorganisms. Five microorganisms were used: Aspergillus oryzae MIM (Microbiologia Industriale Milano) and Rhizopus oryzae CBS $112.07^{\top}$ (Centraalbureau voor Schimmelcultures, Baarn, the Netherlands), R. oryzae CBS 260.28 formerly type of Rhizopus liquefaciens, $R$. oryzae CBS 328.47 formerly type of Rhizopus delemar and $R$. oryzae CBS 391.34 formerly type of Rhizopus javanicus. They were routinely maintained on a solid medium (malt extract $8 \mathrm{~g} / \mathrm{l}$, agar $15 \mathrm{~g} / \mathrm{l}, \mathrm{pH}$ 5.5). Rice hulls were gently furnished by AIRI (Associazione Italiana Risiere Italiane). 
Culture conditions. Submerged fermentations (SMF) were carried out in $750 \mathrm{ml}$ Erlenmeyer flasks containing $100 \mathrm{ml}$ of medium and incubated at $28^{\circ} \mathrm{C}$ for $48 \mathrm{~h}$ on a reciprocal shaker $(100 \mathrm{spm})$. The moulds were grown on media containing a basal medium (BM: Difco yeast extract $1 \mathrm{~g} / \mathrm{l}, \mathrm{K}_{2} \mathrm{HPO}_{4} 1 \mathrm{~g} / \mathrm{l}, \mathrm{MgSO}_{4} \cdot 7 \mathrm{H}_{2} \mathrm{O} 0.2 \mathrm{~g} / \mathrm{l}, \mathrm{pH} 5.8$ ) added with a nitrogen source (peptone, polypeptone, $\mathrm{NaNO}_{3}$ or $\left(\mathrm{NH}_{4}\right)_{2} \mathrm{SO}_{4} 5 \mathrm{~g} / \mathrm{l}$ ) and a carbon source (olive oil or Tween $80,5-20 \mathrm{~g} / \mathrm{l})$. The flasks were inoculated with $10 \mathrm{ml}$ of spore suspension $\left(2 \times 10^{6}\right.$ spores $\left./ \mathrm{ml}\right)$.

Solid state fermentations were performed in $20 \mathrm{~cm}$ Petri dishes. Rice hulls were dried in an air oven at $60{ }^{\circ} \mathrm{C}$ for 48 $\mathrm{h}$, homogenised and sieved to obtain fractions between 1.0 and $2.0 \mathrm{~mm}$ particle size; $10 \mathrm{~g}$ of the obtained rice hulls were impregnated with $13.5 \mathrm{ml}$ of a solution containing: Difco yeast extract $1 \mathrm{~g} / \mathrm{l}, \mathrm{K}_{2} \mathrm{HPO}_{4} 1 \mathrm{~g} / \mathrm{l}, \mathrm{MgSO}_{4} \cdot 7 \mathrm{H}_{2} \mathrm{O} 0.2$ $\mathrm{g} / \mathrm{l}, \mathrm{pH} 5.8$, added with a nitrogen source (polypeptone 5 $\mathrm{g} / \mathrm{l}$ ). Carbon sources (olive oil or Tween 80 ) were separately added at $5-20 \mathrm{~g} / \mathrm{l}$ after sterilisation; the plates were finally inoculated with $1.5 \mathrm{ml}$ of a spore suspension $\left(2 \times 10^{6}\right.$ spores $/ \mathrm{ml}$ ) and incubated at $28{ }^{\circ} \mathrm{C}$ for $48 \mathrm{~h}$; the resulting microbial cultures were lyophilised. The lyophilised cultures were assayed for esterase activity and employed as biocatalyst in esterification trials.

Esterification reactions. Geraniol esterification was performed at $50{ }^{\circ} \mathrm{C}$ under magnetic stirring in $10 \mathrm{ml}$ screw capped test tubes by suspending lyophilised cultures from SSF in $n$-heptane and then adding geraniol $(50 \mathrm{mM})$ and an equimolar amount of the free acids. Control reactions with lyophilised cells coming from SMF were carried out in the same conditions (see Results and Discussion). All the conversion values shown are averages for three different measurements (standard deviation, $\sigma<5 \%$ ).

Esterase activity assays. Esterase activity was assayed using $\alpha$-naphtylacetate, $\alpha$-naphtylbutyrate and $\alpha$-naphtylcaprylate as substrate, measuring the absorbance (wavelength $=560 \mathrm{~nm}$ ) relative to the cromophores originated by reaction of the hydrolysed substrate with Fast Garnet GBS salt (Molinari et al., 2000; Gilham and Lehner, 2005). The mycelia resulting from the SMF growth and the lyophilised cultures obtained with SSF were both suspended in distilled water and homogenised at $4{ }^{\circ} \mathrm{C}$ to obtain a suspension suited for the test.

The solutions of naphtyl derivatives were prepared by adding the substrates $(0.42 \mathrm{mM})$ in $4 \mathrm{ml}$ of ethanol to 96 $\mathrm{ml}$ of buffer solution ( $0.1 \mathrm{M}$ Tris- $\mathrm{HCl}, \mathrm{pH}$ 7.0) containing arabic gum $(10 \mathrm{mg})$ and dioctyl sulfosuccinate sodium salt (AOT, $200 \mathrm{mg}$ ). The reaction mixtures were obtained by adding $1.5 \mathrm{ml}$ of the substrate solution to $100 \mathrm{mg}$ of lyophilised biocatalyst in $0.1 \mathrm{M}$ Tris- $\mathrm{HCl}, \mathrm{pH}$ 7. The enzymatic activity was expressed as unit (U), defined as the amount of biocatalyst which catalysed the transformation of $1 \mu$ mole of substrate in 1 minute at $45^{\circ} \mathrm{C}$.

Analytical methods. Biomass in submerged and solid state cultures was determined by ergosterol content measurements (Desgranges et al., 1991). Alcohol and ester concentrations of the biotransformations in organic solvent were determined by gas-chromatographic (GC) analysis on a Carlo Erba Fractovap GC equipped with a hydrogen flame ionization detector. The column $(3 \times 2000 \mathrm{~mm})$ was packed with Carbowax 1540 (10\% on Chromosorb 80-100 mesh).
The injector temperature was $200^{\circ} \mathrm{C}$. Samples $(0.25 \mathrm{ml})$ were taken at intervals; cells were separated by centrifugation (15000 rpm, $5 \mathrm{~min}$ ) and to the organic phase was added an equal volume of an internal standard solution (1octanol) in $n$-heptane.

\section{RESULTS AND DISCUSSION}

Aspergillus oryzae MIM was preliminarily grown on submerged cultures employing media containing different nitrogen sources (peptone, polypeptone, $\mathrm{NaNO}_{3}$ or $\left.\left(\mathrm{NH}_{4}\right)_{2} \mathrm{SO}_{4}\right)$ and $0.5 \%$ olive oil as main carbon source. The use of $0.5 \%$ polypeptone as nitrogen source gave the highest biomass yields; polypeptone was therefore used as nitrogen source in all the following trials.

It has been previously shown that olive oil and Tween 80 are among the carbon sources suited for producing mycelium of $A$. oryzae MIM with high lipolytic activity (Molinari et al., 2000). Experiments were therefore performed using solid media containing olive oil and Tween 80 as main carbon sources using rice hulls as support; submerged fermentations were also carried out as comparison. The resulting growth values are reported in Table 1.

TABLE 1 - Growth of Aspergillus oryzae MIM on solid-state media (SSF) and submerged cultures (SMF). Growth is expressed as grams of dry mycelium per litre of culture (SMF) or as mg of dry mycelium per gram of inert support.

\begin{tabular}{lccc}
\hline Carbon source & Concentration (\%) & \multicolumn{2}{c}{ Growth } \\
\cline { 3 - 4 } & & SSF & SMF \\
\hline Olive oil & 0.5 & 43 & 4.1 \\
& 1.0 & 52 & 5.3 \\
Tween 80 & 2.0 & 60 & 5.8 \\
& 0.5 & 18 & 2.5 \\
& 1.0 & 24 & 3.2 \\
& 2.0 & 21 & 3.7 \\
\hline
\end{tabular}

The activity of the SMF mycelia and the whole SSF cultures was tested using $\alpha$-naphtyl acetate, butyrate and caprylate as current substrates (see figures $1 \mathrm{~A}$ and $1 \mathrm{~B}$ ).

The highest degree of activity was observed in most of the cases with naphtylbutyrate, while the highest activity towards acetate ester was obtained with mycelia grown using $0.5 \%$ Tween 80 . This approach indicates that the enzyme(s) expressed under these conditions can be considered as "true" esterases (Bornscheuer, 2002).

The whole lyophilised SSF cultures and the lyophilised mycelium from SMF were used as biocatalyst for the synthesis of different geranyl esters (acetate, butyrate and hexanoate). Figure $2 \mathrm{~A}$ reports the acylation of geraniol $(50 \mathrm{mM})$ with equimolar amounts of acetic, butyric and hexanoic acid catalysed by $300 \mathrm{~g} / \mathrm{l}$ of lyophilised SSF culture (corresponding to a concentration of dry mycelium ranging from 5 to $20 \mathrm{~g} / \mathrm{l}$ depending on the growth medium) suspended in heptane at $50{ }^{\circ} \mathrm{C}$. Biotransformations performed with $15 \mathrm{mg} / \mathrm{ml}$ of free lyophilised mycelium are also reported for comparison (see figure 2B). 
A

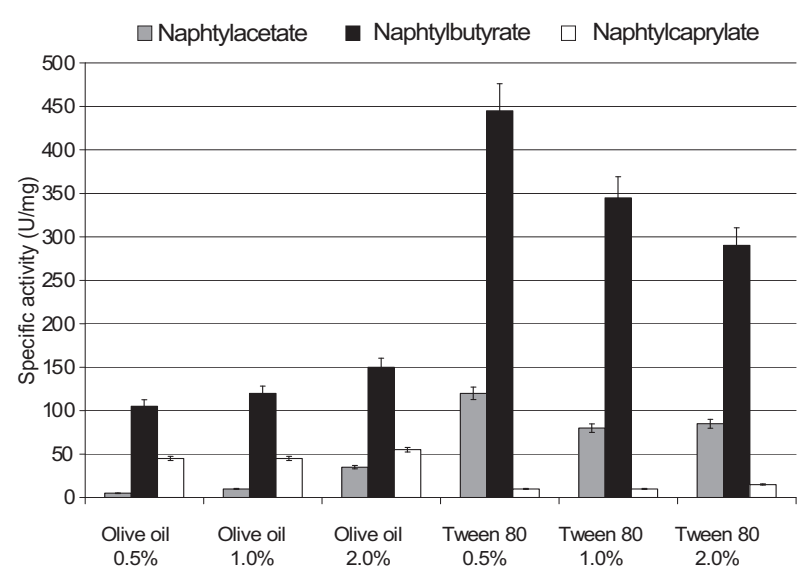

B



FIG. 1 - Hydrolysis of naphtyl-esters with Aspergillus oryzae MIM grown on solid-state media (A) and in submerged cultures (B). Specific activity is expressed as Units per ml of culture (SMF) or as Units per mg of biocatalyst (SSF).

Mycelium grown in SMF cultures with $0.5 \%$ Tween 80 catalysed complete esterification of geranyol with all the carboxylic acids tested, while low activity was found using other C-sources. Molar conversions higher than $70 \%$ were found in the case geranylbutyrate with lyophilised SSF-cultures of $A$. oryzae grown with olive oil or Tween 80 at various concentrations, while the production of geranylacetate and geranylhexanoate was strongly dependent on the carbon source employed; high yields of geranylhexanoate were obtained only using olive oil. Therefore it is likely that different enzymes are expressed depending on the type of fermentation employed.

The good results obtained with $A$. oryzae led us to investigate other four microbial strains, belonging to the species $R$. oryzae (namely $R$. oryzae CBS $112.07, R$. oryzae CBS $260.28, R$. oryzae CBS 328.47 and $R$. oryzae CBS 391.34). These strains had previously shown very effective in promoting the synthesis of esters in organic solvents (Gandolfi et al., 2001). The four strains were grown in SSF using rice hulls as solid support with the medium that afforded the best results with $A$. oryzae (i.e.
$2 \%$ olive oil, polypeptone $5 \mathrm{~g} / \mathrm{l}$ ). A fungal biomass of 51 ( \pm 5.0) $\mathrm{mg}$ of mycelium per gram of solid support was found.

Figure $3 \mathrm{~A}$ reports the acylation of geraniol $(50 \mathrm{mM})$ with equimolar amounts of acetic, butyric and hexanoic acid catalysed by $300 \mathrm{~g} / \mathrm{l}$ of lyophilised SSF culture (corresponding to about $15 \mathrm{mg} / \mathrm{ml}$ of mycelium) suspended in heptane at $50{ }^{\circ} \mathrm{C}$. Biotransformations performed with 15 $\mathrm{mg} / \mathrm{ml}$ of free lyophilised mycelium are also reported for comparison (Fig. 3B).

Lyophilised mycelia of $R$. oryzae grown in SMF furnished higher rates and conversions for acetylation than when grown in SSF. The acylation with butyric acid occurred with similar results using SMF- or SSF-grown mycelium, while the latter was a better catalyst for geranylhexanoate formation, being able to yield more than $80 \%$ molar conversion after 24 hours. It is likely that different sets of enzymes are expressed under different growth conditions. It should be pointed out that $A$. oryzae MIM and $R$. oryzae strains produced also extra-cellular carboxylesterases in SMF, while SSF cultures are directly lyophilised without any downstream operation.
A

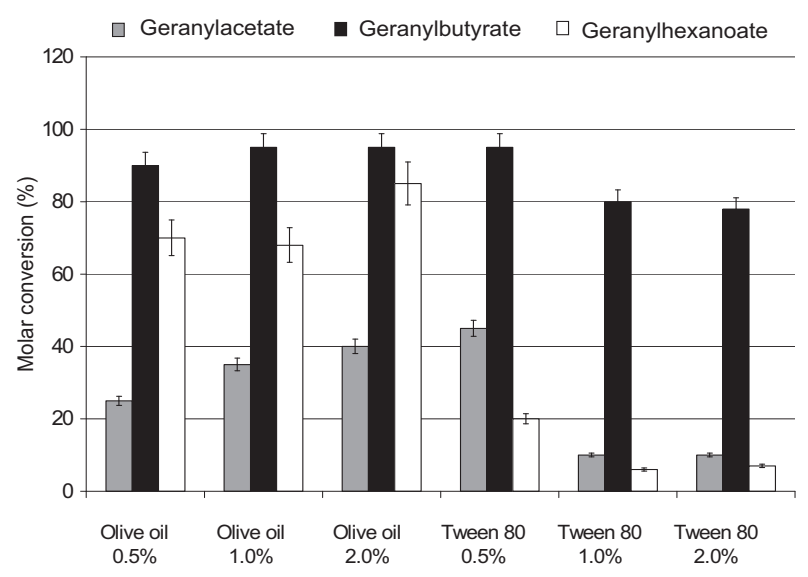

B

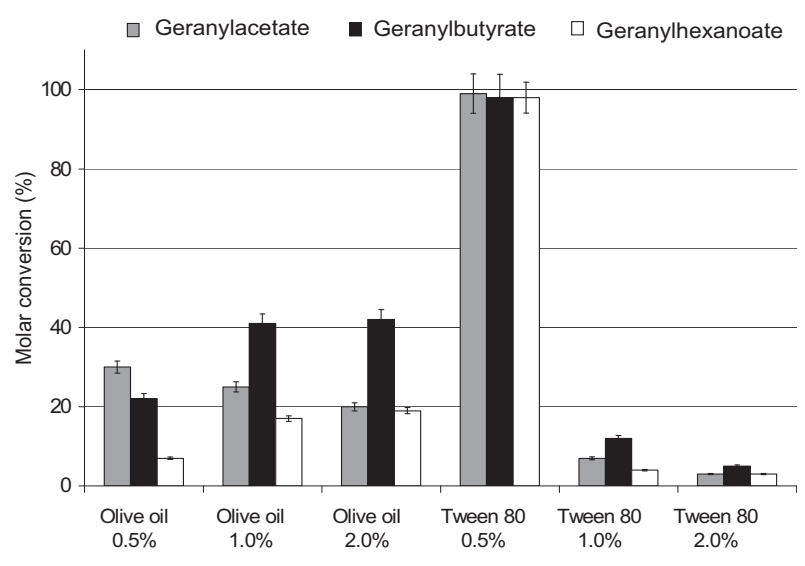

FIG. 2 - Formation of geranyl esters catalysed by Aspergillus oryzae MIM grown on solid-state media (A) and in submerged cultures (B). Molar conversions refer to $24 \mathrm{~h}$ of reaction. 
A



B

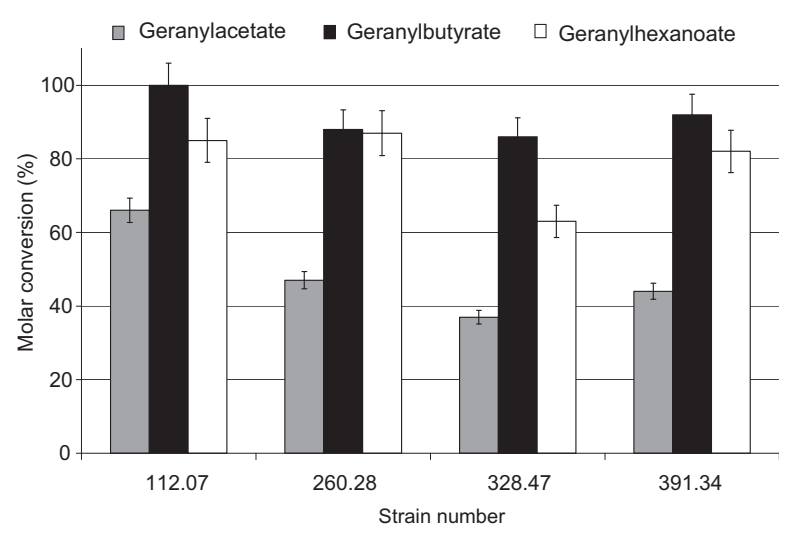

FIG. 3 - Formation of geranyl esters catalysed by Rhizopus oryzae strains grown on solid-state media (A) and in submerged cultures (B). Molar conversions refer to $24 \mathrm{~h}$ of reaction.

In conclusion the esterification in organic solvent catalysed by moulds grown in SSF was studied using $A$. oryzae and $R$. oryzae and compared with the performances of mycelium grown submerged cultures. The whole procedure is very simple and allowed for a stable and efficient catalyst employable in organic solvents for direct esterification of geraniol.

\section{REFERENCES}

Alkan H., Baysal Z., Uyar F., Dogru M. (2007). Production of lipase by a newly isolated Bacillus coagulans under solidstate fermentation using melon wastes. Applied Biochemistry and Biotechnology, 136 (2): 183-192.

Bornscheuer U.T. (2002). Microbial carboxylesterases: classification, properties and application in biocatalysis. FEMS Microbiology Review, 26: 73-81.

Cbristen P., Angeles N., Corzo G., Farres A., Revah S. (1995). Microbial lipase production on a polymeric resin. Biotechnology Techniques, 9: 597-600.

Desgranges C., Vergoignan C., Georges M., Durand A. (1991). Biomass estimation in solid state fermentation. Applied Microbiology Biotechnology, 35: 200-205.

Desgranges C., Vergoignan C., Lereec A., Riba G., Durand A. (1993). Use of solid state fermentation to produce Beauveria bassiana for the biological control of European comborer. Biotechnology Advances, 11: 577-587.

Gandolfi R., Converti A., Pirozzi D., Molinari F. (2001). Efficient and selective microbial esterification with dry mycelium of Rhizopus oryzae. Journal of Biotechnology, 92: 21-26.

Gautam P., Sabu A., Pandey A., Szakacs G., Soccol C.R. (2002). Microbial production of extra-cellular phytase using polystyrene as inert solid support. Bioresource Technology, 83: 229-233.

Gilham D., Lehner R. (2005). Techniques to measure lipase and esterase activity in vitro. Methods, 36: 139-147.

Gutarra M.L.E., Godoy M.G., Castilho L.R., Freire D.M.G. (2007). Inoculum strategies for Penicillium simplicissimum lipase production by solid-state fermentation using a residue from the babassu oil industry. Journal of Chemical Technology and Biotechnology, 82: 313-318.

Gutierrez-Rojas M., Cordova J., Auria R., Revah S., Favela-Toffes E. (1995). Citric acid and polyols production by Aspergillus niger at high glucose concentration in solid state fermentation on insert support. Biotechnology Letters, 17: 219-224.
Hongzhang C., Hui W., Aijun Z., Zuohu L. (2006). Alkaline protease production by solid state fermentation on polyurethane foam. Chemical and Biochemical Engineering Quarterly, 20: 93-97.

Longo M.A., Sanroman M.A. (2006). Production of food aroma compounds: Microbial and enzymatic methodologies. Food Technology Biotechnology, 44: 335-353.

John R.P., Nampoothiri K.M., Pandey A. (2007). Polyurethane foam as an inert carrier for the production of $L(+)$-lactic acid by Lactobacillus casei under solid-state fermentation. Letters in Applied Microbiology, 44 (6): 582-587.

Molinari F., Gandolfi R., Converti A., Zilli M. (2000). Myceliumbound carboxylesterase from Aspergillus oryzae. An efficient catalyst for acetylation in organic solvent. Enzyme and Microbial Technology, 27: 626-630.

Murado M.A., Gonzàlez M. P., Torrado A., Pastrana L. M. (1997). Amylase production by solid state culture of Aspergillus oryzae on polyurethane foams. Some mechanistic approaches from an empirical model. Process Biochemistry (Oxford), 32 (1): 35-42.

Ooijkaas L.P., Weber F.J., Buitelaar R.M., Tramper J., Rinzema A. (2000). Defined media and inert supports: their potential as solid-state fermentation production systems. Trends in Biotechnology, 18: 356-360.

Pandey A., Azmi W., Singh J., Banerejee U.C. (1999). Types of fermentation and factors affecting it. In: Joshi V.H., Pandey A., Eds, Biotechnology: Food Fermentation, Educational Publishers, New Delhi, pp. 383-426.

Pandey A., Soccol C.R., Mitchell D. (2000). New developments in solid state fermentation: bioprocesses and products. Process Biochemistry, 35: 1153-1169.

Qzawa S., Sato K., Endo I. (1996). Repeated batch production of alkaline protease by solid-state fermentation using urethane foam as carriers. Bioprocess Engineering, 14: 63-68.

Silman R.W., Nelsen I.C., Bothast R.J. (1991). Comparison of culture methods for production of Colletotrichum truncatum spores for use as a mycoherbicide. FEMS Microbiology Letters, 79: 69-74.

Silman R.W., Nelsen T.C., Bothast R.J. (1993). Production of Colletotrichum truncatum for use as a mycoherbicide: effects of culture drying and storage on recovery and efficacy. Biotechnology Advances, 11: 561-575.

Zhu Y., Smits J.P., Knol W., Bol J. (1994). A novel solid-state fermentation system using polyurethane foam as inert carrier. Biotechnology Letters, 16: 643-648. 